Disponível em:

http://editora.unoesc.edu.br/index.php/race

Race, Joaçaba, v. 15, n. 3, p. 1143-1168, set./dez. 2016

\title{
CONTABILIDADE DO SETOR PÚBLICO: UM ESTUDO DAS REDES SOCIAIS PUBLICADAS EM PERIÓDICOS INTERNACIONAIS
}

Vania Regina Morás

E-mail: vaniar.moras@gmail.com

Mestre em Ciências Contábeis pelo Programa de Pós-Graduação em Ciências Contábeis da Universidade Regional de Blumenau; Professora do Departamento de Contabilidade da Universidade Regional de Blumenau.

Roberto Carlos Klann

E-mail: rklann@furb.br

Doutor em Ciências Contábeis e Administração pela da Universidade Regional de Blumena; Professor do Programa de Pós-Graduação em Ciências Contábeis da Universidade Regional de Blumenau.

Endereço para contato: Rua Antônio da Veiga, 140, Itoupava Seca, 89012-900, Blumenau, Santa Catarina, Brasil.

Artigo recebido em 13 de maio de 2016. Aceito em 13 de julho de 2016. 
Resumo

No contexto atual, a contabilidade aplicada ao setor público está sendo palco de novas mudanças impostas pela integração dos mercados. Dessa forma, no presente estudo teve-se por objetivo identificar a bibliometria e a sociometria das pesquisas científicas relacionadas ao tema contabilidade pública e contabilidade do setor público, constantes nos artigos científicos indexados na base Science Direct e publicados em periódicos internacionais. Empregou-se metodologia empírico-analítica e longitudinal, por meio de uma pesquisa documental em 20 periódicos internacionais em estudos publicados no período de 1990 a 2014. Filtrou-se pelas expressões public accounting e public sector accounting; a amostra compreendeu 32 artigos. Utilizaram-se as técnicas de análise bibliométrica e análise de redes. Os achados indicam que houve concentração de publicações no período que compreende 2010 a 2014, com média de duas publicações por ano contra a média de uma publicação por ano nos períodos anteriores. Além disso, notou-se uma evolução dos relacionamentos de redes entre os autores. Concluise que o aumento nas publicações no último período analisado pode ter sido influenciado pelo aumento das relações de redes, com maior troca de informações e cooperação entre os pesquisadores da área.

Palavras-chave: Contabilidade pública. Contabilidade aplicada ao setor público. Bibliometria. Sociometria.

Abstract

In the current context, accounting applied to the public sector is the scene of recent changes imposed by market integration. In this study it was aimed to identify bibliometrics and sociometry of scientific research related to the subject public accounting and public sector accounting contained in scientific articles indexed in Science Direct database and published in international journals. An empirical-analytical and longitudinal methodology was developed, through documental research in 20 international business journals in published studies from 1990 to 2014 and filtered by the expressions public accounting and public accounting sector; the sample has consisted of 32 articles. We have used the bibliometric analysis techniques and network analysis. The findings indicate that there was a concentration of publications in the period comprising 2010 to 2014, averaging two publications/year, compared the to average of one publication a year in previous periods. In addition, there has been a trend of network relationships among authors. It is concluded that the publications increase in the last period may have been influenced by the increase in network relationships, with enhanced information exchange and cooperation among researches.

Keywords: Public accounting. Accounting applied to the public sector. Bibliometrics. Sociometry. 


\section{INTRODUÇÃO}

As entidades do setor público se caracterizam por promoverem o bem coletivo e desenvolverem ações sociais, as quais consistem em um conjunto de atividades e bens exercidos ou alocados à disposição da sociedade e visam proporcionar maior grau de bem-estar social e prosperidade pública. Os recursos são oriundos de pessoas físicas ou jurídicas; assim, as entidades governamentais devem prestar contas das ações tomadas e desenvolvidas por meio dos recursos recebidos, com o objetivo de promoverem a transparência pública (SLOMSKI, 2005; LOPES et al., 2010; MACÊDO et al., 2010).

Dessa forma, verificou-se na década de 1990 que o modelo burocrático de administração pública não era mais eficaz para tolerar as mudanças impostas pelas transformações econômicas e sociais, o qual passou a ser criticado pela doutrina da Nova Gestão Pública (New Public Management - NPM), que propôs estruturas na administração pública mais flexíveis e orientadas para o mercado, com maior autonomia para os gestores públicos (PINA; TORRES; ACERETE, 2007; JONES; PENDLEBURY, 2010). Assim, um dos aspectos mais importantes da NPM foi a onda de reformas nos sistemas de informações financeiras, essenciais para a melhoraria da gestão e para a tomada de decisões governamentais (CHRISTIAENS; REYNIERS; ROLLÉ, 2010).

Entre essas mudanças está o aperfeiçoamento dos mecanismos de comunicação com seus usuários, estabelecido pelo processo de harmonização das práticas contábeis internacionais (RIBEIRO; BIZERRA, 2011; ILIE; MIOSE, 2012; TOUDAS; POUTOS; BALIOS, 2013; MELO; PRIETO; ANDRADE, 2014). Christiaens, Reyniers e Rollé (2010) destacam que diante da diversidade de sistemas de informações financeiras governamentais, criou-se a necessidade de unificação e harmonização das normas internacionais de contabilidade no âmbito do setor público. Assim, essa tendência pode ser explicada pela precisão de transparência, eficiência e gerenciamento nessas entidades.

$\mathrm{O}$ atual contexto político e econômico conduz à maior necessidade de geração de informações seguras e confiáveis que possam satisfazer os diversos usuários internos e externos. Portanto, pressupõe-se que a transparência é um meio de acesso à gestão democrática por parte dos usuários e interessados nas informações contábeis e financeiras das entidades governamentais (OLIVEIRA; SILVA; MORAES, 2008). De acordo com Piccoli e Klann (2015), a transparência pública vai além do princípio constitucional da publicidade, pois a informação, além de ser pública, deve ser rele- 
vante, compreensível, segura, acessível e servir como instrumento de acompanhamento da gestão.

Com o objetivo de atender as novas mudanças econômicas e sociais, o International Federation of Accountants (IFAC), com os diversos organismos profissionais de contabilidade ao redor do mundo, criaram o International Public Sector Accounting Standards Board (IPSASB), com o objetivo de desenvolver normas e orientar usuários da contabilidade de entidades do setor público. Dessa forma, desenvolveram-se as International Public Sector Accounting Standards (IPSASs), normas de contabilidade internacional que estabelecem critérios de reconhecimento, mensuração e apresentação, bem como requisitos de divulgação para transações e eventos nas demonstrações financeiras de entidades do setor público (INTERNATIONAL FEDERATION OF ACCOUNTANTS, 2010).

Por sua vez, em 2008, o Brasil iniciou o processo de harmonização, padronização e convergência da contabilidade aplicada ao setor público aos padrões internacionais, sob responsabilidade do Conselho Federal de Contabilidade (CFC), que criou o Comitê Gestor de Convergência. Nesse processo de transição, a contabilidade pública, que estava centrada no controle orçamentário, passou a ter um foco patrimonial, com adoção do regime de competência para as receitas e despesas públicas (CONSELHO FEDERAL DE CONTABILIDADE, 2010; DARÓS; PEREIRA, 2009; RIBEIRO FILHO et al., 2010; VICENTE; MORAIS; PLATT NETO, 2012; SOUZA et al., 2013).

Rossi e Trequattrini (2011) consideram que os pilares da reforma da contabilidade pública são as coordenações das finanças públicas e a harmonização dos sistemas de contabilidade, bem como a implementação de medidas que visam à transparência dos gastos públicos e o controle sobre eles. A adoção das novas normas contábeis permite a consolidação e o monitoramento das contas públicas.

Desse modo, Ilie e Miose (2012) argumentam que as demonstrações contábeis são documentos que fazem parte da prestação de contas das organizações para com as partes interessadas e permitem à sociedade o acompanhamento da execução orçamentária e financeira dos governos. Segundo Grossi e Soverchia (2011), a compilação das demonstrações contábeis consolidadas no setor público deve fornecer uma visão geral do desempenho financeiro e da posição do governo e de todas as organizações que estão sob seu controle.

O foco principal dos estudos observados na área de contabilidade aplicada ao setor público está na internacionalização das normas aplicadas a esse setor, que abrange todos os países de forma gradativa. Em razão das circunstâncias impostas pelas 
transformações econômicas e sociais, esses países buscam aprimorar a evidenciação contábil, a prestação de contas e a transparência pública por meio do processo de harmonização, padronização e convergência às IPSASs (PINA; TORRES; ACERETE, 2007; JONES; PENDLEBURY, 2010; CHRISTIAENS; REYNIERS; ROLLÉ, 2010; ROSSI; TREQUATTRINI, 2011).

Diante do exposto, apresenta-se a problemática: qual a configuração da bibliometria e da sociometria das pesquisas relacionadas ao tema contabilidade pública e contabilidade do setor público constantes nos artigos científicos indexados na base Science Direct, publicados entre 1990 e 2014? O objetivo com este estudo consistiu, então, em identificar a bibliometria e a sociometria das pesquisas científicas relacionadas ao tema contabilidade pública e contabilidade do setor público, constantes nos artigos científicos indexados na base Science Direct e publicados em periódicos internacionais.

No campo teórico internacional, as International Public Sector Accounting Standards (IPSAS) são amplamente discutidas. No Brasil, o fato de municípios, estados e União estarem em processo de mudança em relação às normas contábeis, conforme a Resolução CFC n. 1.128/08, torna ainda esse tema um campo pouco explorado, o que faz surgir a necessidade de realizar pesquisas que buscam identificar o estado da arte sobre essa temática.

Estudos como de Darós e Pereira (2009), Ribeiro Filho et al. (2010), Vicente, Morais e Platt Neto (2012) relatam somente as alterações trazidas pelas Normas Brasileiras de Contabilidade Aplicados ao Setor Público (NBCASPs) e as comparam às legislações anteriores. Lopes et al. (2010) pesquisaram a relação da NBT 16.6 sobre as demonstrações contábeis, analisando as demonstrações exigidas pela Lei n. 4.320/64.

Constata-se que nos últimos anos houve um crescimento do número de publicações nos periódicos nacionais sobre contabilidade pública, bem como nos internacionais no que diz respeito à aplicação da contabilidade por competência e do processo de harmonização. No entanto, estudos cujo objeto tenha sido o Brasil ou de pesquisadores brasileiros publicados em periódicos internacionais ainda são incipientes.

Assim, a relevância do estudo bibliométrico e sociométrico sobre o tema contabilidade pública e contabilidade no setor público na base de Science Direct nas áreas de negócios, gestão e contabilidade e ciências sociais, consiste na sistematização das publicações sobre esse tema. 


\section{REFERENCIAL TEÓRICO}

Neste referencial teórico são abordados temas que embasam conceitualmente a problemática da pesquisa em questão, inicialmente apresentam-se o conceito e o papel do setor público para o bem-estar social, seguidos da contabilidade no setor público com abrangência internacional e nacional.

\subsection{SETOR PÚBLICO}

A expressão setor público faz referência aos governos nacionais, regionais e locais e entidades públicas relacionadas (INTERNATIONAL FEDERATION OF ACCOUNTANTS, 2010). O setor público caracteriza-se por reportar-se ao homem individual, que não consegue mais se defender sozinho e busca por outras pessoas para construir uma sociedade em sua defesa e em defesa de seus bens. Assim, tem por finalidades básicas determinar a segurança, com o objetivo de manter a ordem econômica, política e social e o desenvolvimento, promovendo o bem comum (SILVA, 2011).

Rezende, Slomski e Corrar (2005) consideram importante o papel do setor público no desenvolvimento econômico e social de um país e ressaltam a necessidade de desenvolver novas metodologias que possibilitem mensurar os investimentos sociais e seus impactos na sociedade. Esse setor tem obrigação de fornecer e satisfazer uma ampla gama de necessidades sociais e de expansão contínua, por meio de uma gestão rigorosa dos recursos públicos disponíveis (GRATERON, 1999; SLOMSKI, 2005).

A função primária do setor público é prestar serviços que visam melhorar e manter o bem-estar dos cidadãos, como: programas de bem-estar e policiamento, educação pública, segurança nacional e serviços de defesa (INTERNATIONAL FEDERATION OF ACCOUNTANTS, 2014; INTERNATIONAL PUBLIC SECTOR ACCOUNTANTS STANDARS BOARD, 2014). Dessa forma, o governo e entidades governamentais são responsáveis diante daqueles que fornecem os recursos a prestar contas e informações sobre a aplicação dos recursos. Assim, o Estado tem o dever de prestar contas aos seus cidadãos, evidenciando e tornando públicas suas ações para o bem-estar social (SLOMSKI, 2005).

As mudanças sociais, econômicas, políticas e tecnológicas estão em constante evolução e requerem das entidades a capacidade de adaptação a essas mudanças. As novas tecnologias de informação provocam mudanças no modo de funcionamento do setor público. A disseminação de novas tecnologias pode influenciar de forma significativa a qualidade dos serviços públicos prestados ao cidadão (AMARAL; RON- 
CALIO; ALBERTON, 2013). Nesse contexto, a contabilidade no setor público tem procurado assumir cada vez mais seu papel de destaque no cenário mundial, e sua política de divulgação de informações tem incorporado elementos de responsabilidade social das entidades (REZENDE; SLOMSKI; CORRAR, 2005).

\subsection{CONTABILIDADE NO SETOR PÚBLICO}

A contabilidade pública é o conhecimento especializado da ciência contábil, aplicado no processo de gerar informações conceituais, de princípios e normas contábeis na gestão patrimonial das entidades governamentais, oferecendo à sociedade informações amplas e acessíveis (LIMA; CASTRO, 2012).

A contabilidade pública é aplicada às pessoas jurídicas de Direito Público, que contemplam a União, os estados, o Distrito Federal e os municípios e suas respectivas autarquias e fundações, criadas e mantidas pelo poder público; as empresas públicas e sociedades de economia mista, as quais empregam recursos públicos (LIMA; CASTRO, 2012).

A contabilidade no setor público é um ramo da ciência contábil utilizado pelos gestores como um instrumento de planejamento, controle e elemento indispensável para as informações externas, com o objetivo de fornecer informações precisas para a tomada de decisão. Assim, é fundamental que as informações sejam verdadeiras e coerentes; a falta de veracidade implica a distorção e a omissão da realidade econômica, financeira e patrimonial da administração pública (SOUZA et al., 2013).

Considerando essa necessidade de prestação de contas no âmbito do setor público, a contabilidade pública deve ser entendida como um ramo da contabilidade geral, em que se destaca legalmente a figura do orçamento público, que estima as receitas e fixa as despesas, o planejamento por meio do Plano Diretor, Plano Plurianual, Lei de Diretrizes Orçamentárias e Lei do Orçamento. A gestão do patrimônio público não está voltada à obtenção de lucro financeiro, mas, sim, para o denominado lucro social, que será distribuído em benefício da sociedade (ANDRADE, 2012).

Ferreira e Marchesini (2011, p. 1) destacam que “[...] a contabilidade pública é um ramo da aplicação da contabilidade geral que tem por objetivo evidenciar o patrimônio público e suas modificações.” Para isso, é necessário o cumprimento dos princípios fundamentais de contabilidade e da legislação aplicável ao setor público. Chan (2006) argumenta que a contabilidade pública se refere aos sistemas de informação financeira de um governo e às práticas de divulgação dessas informações. O 
seu desenvolvimento resulta da disponibilização das informações financeiras e sua transparência.

Por fim, a Resolução CFC n. 1.128/08, que aprovou a NBC T 16.1 Conceituação, Objetivo e Campo de Aplicação da Contabilidade Aplicada ao Setor Público, posteriormente alterada pela Resolução CFC n. 1.437/13, determina que a "Contabilidade Aplicada ao Setor Público é o ramo da ciência contábil que aplica, no processo de geração de informações, os Princípios de Contabilidade e as normas contábeis direcionadas ao controle patrimonial de entidades do setor público.” (CONSELHO FEDERAL DE CONTABILIDADE, 2008).

O objetivo da contabilidade pública é fornecer informações aos usuários sobre os resultados alcançados para o processo de tomada de decisão, prestação de contas e instrumento de controle social. Tem por objeto de estudo o patrimônio público (RESOLUÇÃO CFC n. 1.128/08). Dessa forma, cabe às entidades do setor público desenvolverem os relatórios financeiros para responder às necessidades de informações dos usuários, destinatários de serviços e provedores de recursos. Diante da necessidade de prestação de contas à sociedade, em âmbito mundial, a contabilidade aplicada ao setor público em diversos países vem aderindo ao processo de internacionalização das normas (INTERNATIONAL FEDERATION OF ACCOUNTANTS, 2014).

\subsection{INTERNATIONAL PUBLIC SECTOR ACCOUNTING STANDARDS (IPSAS)}

As IPSASs são normas de contabilidade internacional que estabelecem critérios de reconhecimento, mensuração e apresentação, bem como requisitos de divulgação para transações e eventos nas demonstrações financeiras de entidades do setor público (ROSSI; TREQUATTRINI, 2011). Dessa forma, a introdução das IPSASs no setor público impulsiona os países a modificarem suas políticas e procedimentos contábeis. Cada jurisdição possui suas próprias regras e regulamentos contábeis, porém precisam se adequar ao novo modelo de contabilidade pública (CHAN, 2006). A tendência à harmonização, padronização e convergência internacional das normas contábeis aparenta ser uma necessidade imposta pela integração dos mercados (INTERNATIONAL FEDERATION OF ACCOUNTANTS, 2010).

A padronização das normas internacionais de contabilidade do setor público tende a gerar benefícios significativos no intuito de harmonizar as informações financeiras de forma coerente e comparável entre as jurisdições. Portanto, cabe às IPSASs desempenhar um papel fundamental no sentido de permitir que sua adoção pelos governos melhorará a qualidade e a comparabilidade das informações financeiras 
das entidades do setor público (INTERNATIONAL FEDERATION OF ACCOUNTANTS, 2003). Assim, a harmonização dos sistemas de contabilidade nos diversos níveis de governo é essencial para aumentar a transparência nos processos de decisão e coordenação da gestão pública (ROSSI; TREQUATTRINI, 2011).

Os objetivos das IPSASs são promover uma maior responsabilidade na prestação de contas por parte dos governos dos países e, consequentemente, demonstrar qualidade e confiabilidade contábil por meio dos relatórios financeiros, visando melhorar a gestão financeira e harmonizar as informações financeiras em âmbito internacional (CHAN, 2006). De certa forma, as IPSASs dispõem de uma contabilidade patrimonial integral que solicita o registro de todos os itens de bens, direitos e obrigações, bem como do patrimônio líquido da entidade contabilizada, estabelecendo o regime de competência no reconhecimento de receitas e despesas (CONSELHO FEDERAL DE CONTABILIDADE, 2010; MORAIS; PLATT NETO, 2011). Assim, a partir de 2003 a 2014, o IPSASB desenvolveu um conjunto de 32 normas denominadas IPSASs, as quais estão publicadas no Handbook of International Public Sector Accounting Pronouncements Vol. 1 e Vol. 2 da Edição de 2014 (INTERNATIONAL FEDERATION OF ACCOUNTANTS, 2014).

Portanto, o desenvolvimento econômico mundial e a necessidade de transparência nas informações divulgadas, sejam elas oriundas do setor privado, sejam do setor público, contribuíram para a discussão sobre a harmonização das normas de contabilidade aplicadas ao setor público também em âmbito nacional. Diante desse contexto, o setor público brasileiro está sendo pauta de discussões e debates sobre os desafios apresentados pelo processo de convergência às normas internacionais (MACÊDO; KLANN, 2014).

\subsection{NORMAS BRASILEIRAS DE CONTABILIDADE APLICADAS AO SETOR PÚBLICO (NBCASPS)}

Em decorrência da expansão econômica, a administração pública brasileira necessita de maior controle e evidenciação dos recursos públicos para fornecer informações contábeis precisas e transparentes em relação aos demais países. Diante dessa necessidade, é imprescindível aderir ao processo de convergência das práticas contábeis brasileiras aplicadas ao setor público com as normas internacionais de contabilidade desenvolvidas para esse setor (MENDONÇA; MACHADO, 2010).

Assim, com o objetivo de promover as adequações necessárias à convergência aos padrões internacionais de contabilidade, o Tesouro Nacional promoveu mais uma 
ação do seu Planejamento Estratégico em virtude da padronização dos procedimentos contábeis entre entes da Federação (União, Estados, Distrito Federal e Municípios), promovendo a consolidação das contas públicas e a sua convergência metodológica e conceitual às Normas Internacionais e às Normas Brasileiras de Contabilidade Aplicadas ao Setor Público, conforme a Portaria MF n. 184/2008 e o Decreto n. 6.976/2009.

O CFC, na qualidade de órgão regulador das práticas contábeis e também membro do IFAC, designou um grupo de trabalho para desenvolver as primeiras Normas Brasileiras de Contabilidade Aplicadas ao Setor Público, de acordo com as IPSASs. As normas elaboradas foram submetidas a audiências públicas e discutidas com apresentações de sugestões, o que resultou, no final de 2008, na publicação de 10 normas (DARÓS; PEREIRA, 2009; LIMA; GUEDES; SANTANA, 2009). Em novembro de 2011, o CFC editou a $11^{\text {a }}$ norma, conforme a Resolução CFC n. 1.366/11, que aprovou a NBC T 16.11 sobre sistemas de informações de custos no setor público.

A edição das Normas Brasileiras de Contabilidade Aplicadas ao Setor Público (NBCASPs) procura destacar a importância dos aspectos patrimoniais no setor público, contemplando os aspectos orçamentários, complementada pelas alterações na estrutura do Plano de Contas do Setor Público, na reestruturação dos anexos da Lei Complementar n. 4.320/64 em convergência com as normas internacionais (CASTRO, 2013).

As NBCASPs surgiram com a finalidade de harmonizar as práticas contábeis públicas brasileiras às normas internacionais de contabilidade, a fim de padronizar a contabilidade da União, dos Estados e dos Municípios. Esse processo está normatizado por meio das Resoluções CFC n. 1.128 a 1.137, de 21 de novembro de 2008, e da Resolução n. 1.268, de 10 de dezembro de 2009, que altera, inclui e exclui itens das NBCASPs 16.1, 16.2 e 16.6 (CONSELHO FEDERAL DE CONTABILIDADE, 2014). As NBCASPs trouxeram mudanças ao setor público e impactaram as atividades operacionais dos contadores públicos. Destaca-se o princípio da competência como uma das mudanças trazidas pelas normas, além de outras que tratam das transações com o setor público, depreciação, planejamento, registro contábil e demonstrações contábeis (PICCOLI; KLANN, 2014).

A nova contabilidade pública busca aprimorar e fortalecer as relações do Estado com a sociedade, à medida que os governos devem assegurar que informações objetivas, confiáveis, íntegras, seguras e relevantes sejam disponibilizadas de forma acessível e de fácil compreensão, que não apenas contemplem a obrigação do governo em prestar contas, mas também contribuam para o aperfeiçoamento da gestão pública (SLOMSKI, 2005). 
Para tanto, a Secretária do Tesouro Nacional (STN) (2015) iniciou um trabalho em parceria com a União, os estados, o Distrito Federal e os municípios visando à implantação do Plano de Contas Aplicado ao Setor Público (PCASP) em toda a Federação a partir de 2010. No final de 2013 a STN publicou a Portaria n. 634, de 19 de novembro de 2013, em que estabeleceu como prazo final para a implantação do PCASP a data de 31 de dezembro de 2014, com vigência para o exercício de 2015. Diante do exposto, infere-se que a contabilidade no setor público brasileiro ainda está se adequando à convergência às normas internacionais de contabilidade aplicadas ao setor público.

\section{ASPECTOS METODOLÓGICOS}

Com base no objetivo proposto, o procedimento metodológico adotado foi concebido como uma pesquisa empírico-analítica, sendo realizada uma pesquisa descritiva com perspectiva longitudinal, por meio de uma pesquisa documental (CRESWELL, 1994). Utilizaram-se as técnicas de análise bibliométrica e de redes para comparar as principais características demonstradas pelas redes de autoria e coautoria.

A pesquisa bibliométrica proporciona estudar os aspectos quantitativos, da disseminação do conhecimento e a utilização da informação registrada. O estudo sociométrico busca relacionar os autores que fazem parte da mesma rede de autoria e coautoria (MACIAS-CHAPULA, 1998; WASSERMAN; GALASKIEWICZ, 1994). Entender a sociometria, ou seja, a formação de redes para a produção de pesquisas e artigos entre autores e coautores, define-se como uma forma de analisar e compreender a estrutura de um campo do conhecimento. A bibliometria, nas pesquisas científicas, busca mapear as informações e, consequentemente, analisar a produtividade dos autores e das áreas do conhecimento (CRANE, 1972). Pinto et al. (2007) destacam que as redes sociais se constituem em uma representação de frequência científica, apresentadas em mapas a partir da semelhança de autores, tópicos ou instituições científicas. Para fortalecer os aspectos quantitativos das redes, é importante agregar estudos métricos com vários aspectos das relações.

Dessa forma, neste estudo buscou-se a identificação e a compilação dos trabalhos associados ao tema contabilidade pública e contabilidade do setor público disponibilizados na base de dados Science Direct, no período de 1990 a 2014, por meio de uma abordagem quantitativa. A acessibilidade às bases de dados resultou na escolha da base Science Direct. Ressalta-se, ainda, que a escolha do período aconteceu 
em virtude do surgimento da New Public Management, que resultou em uma onda de reformas nos sistemas de informações financeiras, essenciais para melhorar a gestão e a tomada de decisões governamentais, ocasionando a disseminação das informações no setor público (CHRISTIAENS; REYNIERS; ROLLÉ, 2010). Desse modo, infere-se que a partir da NPM os pesquisadores tenham dado mais importância aos temas relacionados à contabilidade aplicada ao setor público.

Para a coleta dos dados, filtraram-se as expressões Public Accounting e $\mathrm{Pu}$ blic Sector Accounting, no título, no resumo e nas palavras-chave dos artigos. A busca resultou em 156 artigos publicados em 20 periódicos diferentes, conforme segue: Accounting, Organizations and Society, Advances in Accounting, Advances in International Accounting, Critical Perspectives on Accounting, Industrial Marketing Management, International Journal of Accounting Information Systems, Journal of Accounting and Economics, Journal of Accounting and Public Policy, Journal of Accounting Education, Journal of Business Research, Journal of Corporate Finance; Journal of Financial Economics; Journal of International Accounting, Auditing and Taxation, Management Accounting Research, Omega, Scandinavian Journal of Management, Technovation, The British Accounting Review, Tékhne - Review of Applied Management Studies, e The International Journal of Accounting.

Dos 156 artigos obtidos, 124 não estavam relacionados ao tema, sendo a amostra final composta por 32 artigos. Para identificar a abordagem principal dos artigos, realizou-se a análise de conteúdo no título, no resumo e nas palavras-chave. As análises bibliométrica e de redes seguiram o levantamento de dados dos itens: período de publicação dos artigos (1990 a 2014), periódico, rede social no período, autores que mais produzem e com maior número de laços, indicadores da rede, entre autores. Para gerar as figuras representativas da estrutura da rede e seus indicadores empregou-se o software UCINET® 6.

\section{DESCRIÇÃO E ANÁLISE DOS RESULTADOS}

Nesta seção são apresentadas a descrição e a análise dos resultados referentes ao estudo elaborado. Na Tabela 1 destaca-se o número de artigos analisados por periódico e por período. 
Tabela 1 - Número de artigos sobre contabilidade pública e contabilidade do setor público publicados em cada periódico por período

\begin{tabular}{|c|c|c|c|c|}
\hline Periódico & $\begin{array}{c}1990 \text { a } \\
1999\end{array}$ & 2000 a 2009 & $\begin{array}{c}2010 \text { a } \\
2014\end{array}$ & Total \\
\hline Accounting, Organizations and Society & 2 & 1 & & 3 \\
\hline Accounting Forum & & & 1 & 1 \\
\hline Advances in Accounting & & 1 & & 1 \\
\hline Critical Perspectives on Accounting & 1 & 3 & 4 & 8 \\
\hline Journal of Accounting Education & 2 & 1 & & 3 \\
\hline $\begin{array}{l}\text { Journal of International Accounting Auditing } \\
\text { \& Taxation }\end{array}$ & 2 & & & 2 \\
\hline Journal of Accounting and Public Policy & & 1 & & 1 \\
\hline Management Accounting Research & & 1 & 2 & 3 \\
\hline Procedia - Social and Behavioral Sciences & & & 1 & 1 \\
\hline $\begin{array}{l}\text { Revista de Contabilidade-Spanish Accounting } \\
\text { Review }\end{array}$ & & & 1 & 1 \\
\hline The British Accounting Rewiew & 2 & & 2 & 4 \\
\hline The International Journal of Accounting & 1 & 1 & & 2 \\
\hline $\begin{array}{l}\text { TÉKHNE - Review of Applied Management } \\
\text { Studies }\end{array}$ & & & 2 & 2 \\
\hline \multirow{2}{*}{ Total } & 10 & 9 & 13 & 32 \\
\hline & $31 \%$ & $28 \%$ & $41 \%$ & $100 \%$ \\
\hline
\end{tabular}

Fonte: os autores.

Conforme a Tabela 1, o maior número de publicação compreende o período de 2010 a 2014, com 13 publicações. No entanto, analisando os três períodos (1990 a 2014), observa-se que os números de artigos publicados são semelhantes. O periódico que mais publicou artigos sobre o tema foi o Critical Perspectives on Accounting, com oito artigos. Assim, 32 artigos constam em 13 periódicos diferentes.

Na Tabela 2 apresenta-se o número de coautorias por artigo publicado; para essa demonstração, manteve-se a segregação por período.

Tabela 2 - Número de coautorias por artigo

\begin{tabular}{|c|c|c|c|c|c|c|c|c|c|c|}
\hline Período & $\begin{array}{c}1 \text { Au- } \\
\text { tor }\end{array}$ & $\%$ & $\begin{array}{l}2 \mathrm{Au}- \\
\text { tores }\end{array}$ & $\%$ & $\begin{array}{c}3 \text { Au- } \\
\text { tores }\end{array}$ & $\%$ & $\begin{array}{l}3 \mathrm{Au}- \\
\text { tores }\end{array}$ & $\%$ & $\begin{array}{l}\text { To- } \\
\text { tal }\end{array}$ & $\%$ \\
\hline 1990 a 1999 & 6 & $55 \%$ & 4 & $29 \%$ & 0 & $0 \%$ & 0 & $0 \%$ & 10 & $31 \%$ \\
\hline 2000 а 2009 & 2 & $18 \%$ & 3 & $21 \%$ & 3 & $60 \%$ & 1 & $50 \%$ & 9 & $28 \%$ \\
\hline 2010 a 2014 & 3 & $27 \%$ & 7 & $50 \%$ & 2 & $40 \%$ & 1 & $50 \%$ & 13 & $41 \%$ \\
\hline Total & 11 & $34 \%$ & 14 & $44 \%$ & 5 & $16 \%$ & 2 & $6 \%$ & 32 & $100 \%$ \\
\hline
\end{tabular}

Fonte: os autores. 
Destaca-se na Tabela 2 que os autores dos artigos analisados preferem realizar publicações em duplas, totalizando 14 artigos. Observa-se, ainda, que 11 publicações são individuais, e cinco artigos são publicados em trio; somente dois artigos apresentam mais de três autores. Essa configuração das coautorias apresentadas na Tabela 2 pode representar certa resistência dos autores dessa temática em trabalhar em redes.

A Figura 1 demonstra as redes de coautoria por período (1990 a 1999). Cada nó (bolinhas) representa uma publicação, cujos laços (setas) expressam relações de autoria. Neste estudo, os nós são interpretados como pesquisadores que proporcionam conhecimento à pesquisa, cada nó representa uma função de ligação, já o laço representa a colaboração entre dois ou mais pesquisadores.

Figura 1 - Rede de coautoria por período (1990 a 1999)
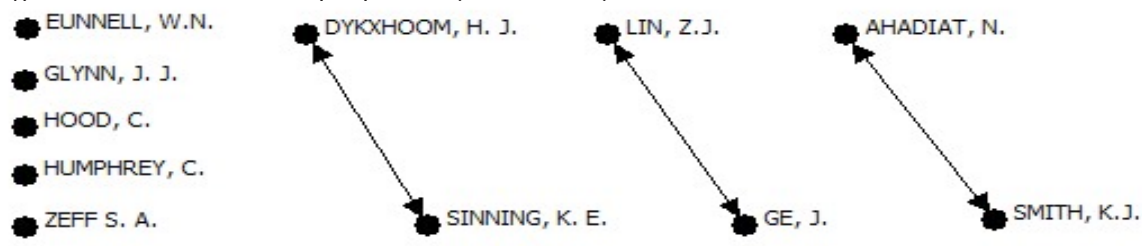

Fonte: os autores.

Conforme a Figura 1, o período que compreende 1990 a 1999 representa a segunda maior concentração de estudos publicados sobre contabilidade pública e contabilidade do setor público, com 10 publicações, representando 34\% das publicações analisadas. Dessa forma, a caracterização da rede de autoria distribuiu-se em cinco publicações independentes, representando um laço (EUNNELL; GLYNN, 1990; HOOD, 2000; ZEFF, 1995; HUMPHREY, 1992) e três publicações díades, representando dois laços entre autores (GE; LIN; 1993; DYKXHOORN; SINNING, 1992, 1996; SMITH; AHADIAT, 1995).

A formação de laços entre pesquisadores conectados em rede favorece as trocas de informações (MARTES et al., 2006).

Na sequência, apresenta-se o comportamento da rede de coautoria para o período de 2000 a 2009. 
Figura 2 - Rede de coautoria por período (2000 a 2009)

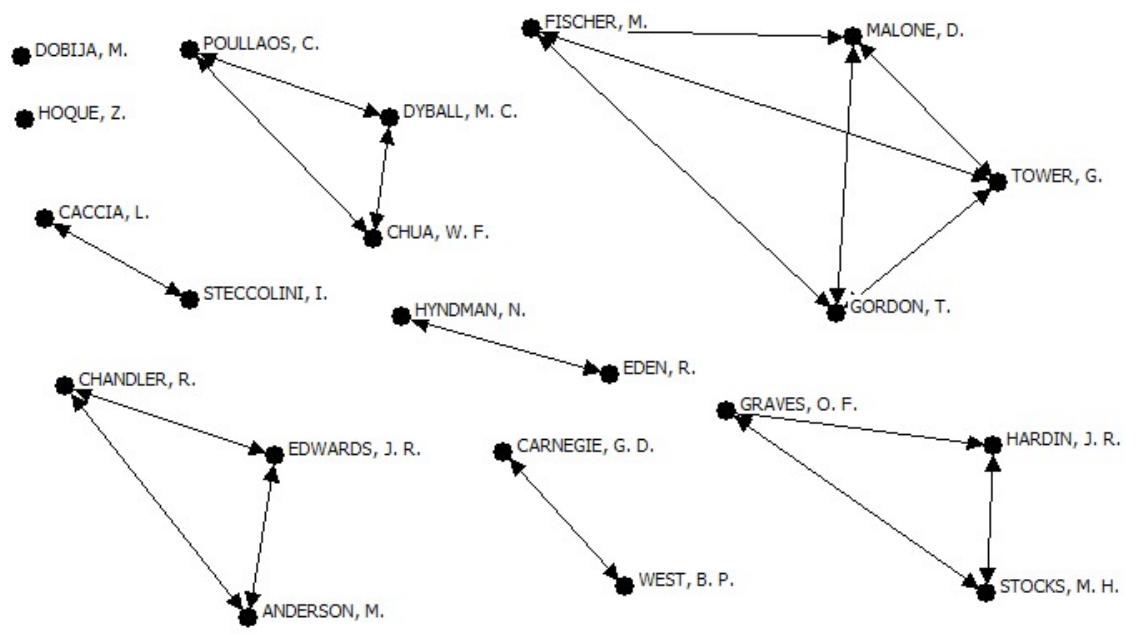

Fonte: os autores.

Observa-se, na Figura 2, que no período de 2000 a 2009 ocorreram 10 publicações, sendo duas publicações independentes, três em díades, três em tríades, e uma publicação representada por quatro autores, ocasionando quatro relações de laços. Os autores que publicaram artigos sozinhos, como Dobija (2000) e Hoque (2002), representam apenas um laço. As publicações entre dois ou mais autores representam dois ou mais laços de relações (CACCIA; STECCOLINI, 2006; HYNDMAN; EDEN, 2000; CARNEGIE; WEST, 2005; EDWARDS; ANDERSON; CHANDLER, 2007).

Destaca-se que no período entre 2000 e 2009 o número de laços aumentou consideravelmente em relação ao período de 1990 a 1999, embora a quantidade de artigos tenha sido semelhante (9 e 10, respectivamente). Tal fato pode representar uma evolução dos relacionamentos de redes entre os autores que tratam do tema, o que também pode contribuir para a melhoria do conhecimento sobre esse campo. Martes et al. (2006) destacam que os relacionamentos são constitutivos da natureza humana e que eles são elementos definidores da identidade dos atores sociais, ou seja, a necessidade de adquirir informações sobre determinado campo leva os autores a relacionarem-se entre si.

Na Figura 3 apresenta-se o último período analisado, compreendido entre 2010 e 2014. 
Figura 3 - Rede de coautoria por período (2010 a 2014)

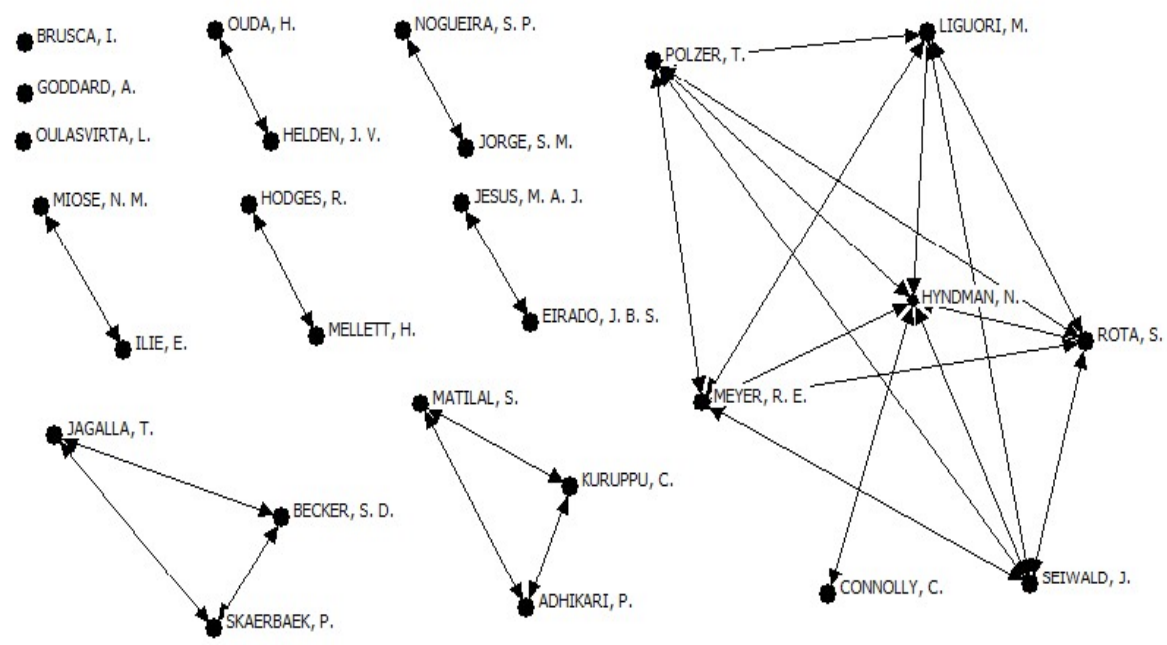

Fonte: os autores.

Neste período de 2010 a 2014, em um espaço de cinco anos foram realizadas 13 publicações, permanecendo estável em relação aos dois períodos anteriores analisados com o tema contabilidade pública e contabilidade do setor público. A relação entre autores representa a seguinte configuração: três publicações independentes, sete publicações constando dois autores, duas publicações com três autorias e, por fim, uma publicação com mais de três autorias.

Observa-se que o período representado na Figura 3 apresenta maior número de conexões (seis conexões) entre os artigos analisados. Nesse período destaca-se também a formação de redes entre duas autorias como: Helden e Ouda (2013), Mellett e Hodges (2012); Ilie e Miose (2012); Jorge e Nogueira (2012) e Eirado e Jesus (2012).

Tal fato confirma a tendência observada no período anterior (2000 a 2009), de crescimento dos relacionamentos de redes entre autores do tema. Neste último período analisado as redes se tornaram ainda maiores do que no período anterior, aumentando a troca de informações entre os autores.

A seguir apresenta-se o Quadro 1, em que se destaca o número de citações por artigo publicado. Optou-se por elencar os 10 artigos com mais citações com base no Google Acadêmico. Entre os estudos pesquisados em relação ao número de citações, observa-se que o estudo de Hood (1995) pode ser considerado o de maior destaque na amostra, com 2.526 citações, destacando-se com o tema sobre a New Public Management. Os demais estudos, como o de Carnegie e West (2005), segunda publicação 
mais citada com 101 citações, Caccia e Steccolini (2006) com 70 citações e Gordon et al. (2002), citado 65 vezes, apresentam volume de citações significativamente inferior ao trabalho de Hood (1995).

Quadro 1 - Número de citações por artigos

\begin{tabular}{|c|c|c|c|c|}
\hline Título & Autor & Co-Autores & Ano & Citações \\
\hline $\begin{array}{l}\text { The "New Public Management" in } \\
\text { the 1980: Variations on a Theme }\end{array}$ & Hood, C. & & 1995 & 2.526 \\
\hline $\begin{array}{l}\text { Making accounting accountable in } \\
\text { the public sector }\end{array}$ & $\begin{array}{l}\text { Carnegie, } \\
\text { G. D. }\end{array}$ & West, B. P. & 2005 & 101 \\
\hline $\begin{array}{l}\text { Accounting change in Italian local } \\
\text { governments: What's beyond mana- } \\
\text { gerial fashion? }\end{array}$ & Caccia, L. & Steccolini, I. & 2006 & 70 \\
\hline $\begin{array}{l}\text { A comparative empirical examina- } \\
\text { tion of extent of disclosure by priva- } \\
\text { te and public colleges and universi- } \\
\text { ties in the United States }\end{array}$ & Gordon, T. & $\begin{array}{l}\text { Fischer, M.; Malone, } \\
\text { D.; Tower, G. }\end{array}$ & 2002 & 65 \\
\hline $\begin{array}{l}\text { A study of the coordination of mis- } \\
\text { sion, objectives and targets in U.K. } \\
\text { executive agencies }\end{array}$ & Hyndman, N. & Eden R. & 2000 & 48 \\
\hline $\begin{array}{l}\text { Claiming a jurisdiction for the "Pu- } \\
\text { blic Accountant" in England prior to } \\
\text { organizational fusion }\end{array}$ & Edwards, J. R. & $\begin{array}{l}\text { Anderson, M.; Chan- } \\
\text { dler, R. A. }\end{array}$ & 2007 & 40 \\
\hline $\begin{array}{l}\text { Accounting and empire: Professio- } \\
\text { nalization-as-resistance The case of } \\
\text { Philippines }\end{array}$ & Dyball, M. C. & $\begin{array}{l}\text { Poullaos, C.; Chua, } \\
\text { W. F. }\end{array}$ & 2007 & 32 \\
\hline $\begin{array}{l}\text { Reflecting on attempts to develop a } \\
\text { financial management information } \\
\text { system for the probation servisse in } \\
\text { England and wales: some observa- } \\
\text { tions on the relationship between the } \\
\text { claims of accounting and its practice }\end{array}$ & Humphrey, C. & & 1994 & 30 \\
\hline $\begin{array}{l}\text { Accruals accounting in the public } \\
\text { sector: A road not Always taken }\end{array}$ & Hyndman, N. & Connolly, C. & 2011 & 30 \\
\hline $\begin{array}{l}\text { Contemporary public sector ac- } \\
\text { counting research - an international } \\
\text { comparison of journal papers }\end{array}$ & Goddard, A. & & 2010 & 26 \\
\hline
\end{tabular}

Fonte: os autores.

Dessa forma, considerando, ainda, que se trata de um dos estudos mais “antigos” da amostra, infere-se que o estudo de Hood (1995) pode ser considerado um 
dos mais influentes na literatura sobre NPM, sendo importante fonte para as pesquisas sobre o tema.

Outra informação presente no Quadro 1 é que os anos 2005 a 2007 podem ser considerados os mais produtivos entre os artigos com maiores citações, compreendendo $40 \%$ dos 10 artigos mais citados. Por outro lado, não foram encontrados estudos relevantes (com mais citações) nos últimos quatros anos (2012 a 2015), o que pode indicar uma tendência de queda na discussão dessa temática em âmbito internacional.

Esse resultado vai de encontro ao observado na Tabela 1, que aponta para um maior número de estudos no período de 2010 a 2014.

\section{CONSIDERAÇÕES FINAIS}

Neste estudo objetivou-se identificar a bibliometria e a sociometria das pesquisas científicas relacionadas ao tema contabilidade pública e contabilidade do setor público, constantes nos artigos científicos indexados na base Science Direct e publicados em periódicos internacionais. Para tal, realizou-se pesquisa bibliométrica e sociométrica com metodologia empírico-analítica em 20 periódicos internacionais. Para a seleção dos artigos, filtraram-se as expressões public accounting e public sector accounting no título, no resumo e nas palavras-chave dos artigos, resultando em uma amostra de 32 artigos. Os resultados da pesquisa demonstram que o número de artigos publicados e a cooperação entre as redes apresentaram uma evolução no decorrer de 20 anos, comparando-se os três períodos (1990-1999, 2000-2009, 2010-2014).

Observou-se que no último período analisado (2010 a 2014) as publicações são relativamente maiores em relação ao primeiro e ao segundo período de análise. Conclui-se que tal resultado pode ter sido influenciado pelo desenvolvimento das redes entre os autores, que pode ter contribuído para a troca de informações e a cooperação entre os pesquisadores e, consequentemente, em razão das mudanças que estão ocorrendo na contabilidade aplicada ao setor público a partir da introdução das IPSASs, em nível internacional. No entanto, de maneira geral, pode-se dizer que ainda há baixa densidade das redes de coautoria, pois os autores de artigos relacionados ao tema preferem publicações isoladas, em díades e tríades.

Conclui-se, ainda, que a baixa densidade das redes de coautoria ocorre em virtude de um número inferior de pesquisadores na temática contabilidade aplicada ao setor público, os quais estão localizados em diferentes pontos e não se baseiam na troca de informações para fortalecimento das pesquisas. Além disso, cabe salientar que os artigos mais citados tratam dos temas New Public Management e contabilidade 
aplicada ao setor público, em especial, nos países europeus, havendo incipiência de artigos brasileiros publicados em periódicos da base de dados Science Direct. A partir dos resultados encontrados, destaca-se a necessidade de ampliar o número de publicações, já que em 20 anos foram encontradas 32 publicações relacionadas ao tema.

Destaca-se como contribuição deste estudo a detecção de redes de cooperação entre os autores da temática, que pode auxiliar futuras pesquisas na área a identificarem autores de referência que trabalham em conjunto, cujos estudos podem indicar linhas de pesquisa específicas. Além disso, o levantamento dos estudos mais citados pode indicar os artigos seminais do tema que devem ser utilizados no referencial teórico para fundamentar futuras pesquisas.

Como limitações do estudo, pode-se citar a utilização de uma única base para realizar a pesquisa dos artigos (Science Direct), bem como o fato de não ter sido realizada uma análise a respeito do conteúdo dos estudos. Para as próximas pesquisas, sugere-se a utilização do mesmo tema com busca em outras bases.

\section{REFERÊNCIAS}

ADHIKARI, P.; KURUPPU, C.; MATILAL, S. Dissemination and institutionalization of public sector accounting reforms in less developed countries: a comparative study of the Nepalese and Sri Lankan central governments. Accounting Forum, Elsevier, p. 213-230, 2013.

AMARAL, E.; RONCALIO, M. P.; ALBERTON, L. Adoção do enfoque patrimonial, previsto nas normas brasileiras de contabilidade aplicadas ao setor público, em municípios de Santa Catarina. Gestão Contemporânea, n. 13, 2013.

ANDRADE, N. A. Contabilidade pública na gestão municipal. 4. ed. São Paulo: Atlas, 2012.

BECKER, S. D.; JAGALLA, T.; SKÆRBÆK, P. The translation of accrual accounting and budgeting and the reconfiguration of public sector accountants' identities. Critical Perspectives on Accounting, v. 25, i. 4, p. 324-338, 2013.

BRASIL. Decreto n. 6.976, de 07 de outubro de 2009. Dispõe sobre o sistema de contabilidade federal e dá outras providências. Diário Oficial da União, Brasília, DF, 07 out. 2009. Disponível em: <http://www.planalto.gov.br/ccivil_03/_Ato20072010/2009/Decreto/D6976.htm>. Acesso em: 10 set. 2014. 
BRUSCA, I. Treinta años de investigación en contabilidad y gestión pública en España. Revista de contabilidad, v. 13, n. 2, p. 175-209, 2010.

CACCIA, L.; STECCOLINI, I. Accounting change in Italian local governments: what's beyond managerial fashion? Critical Perspectives on Accounting, v. 17, i. 2, p. 154-174, 2006.

CARNEGIE, G. D.; WEST, B. P. Making accounting accountable in the public sector. Critical perspectives on accounting, v. 16, i. 7, p. 905-928, 2005. CASTRO, D. P. Auditoria, contabilidade e controle interno no setor público: integração das áreas do ciclo de gestão: planejamento, orçamento, finanças, contabilidade e auditoria e organizações dos controles internos, como suporte à governança corporativa. 5. ed. São Paulo: Atlas, 2013.

CHAN, J. L. IPSAS and government accounting reform in developing countries. Accounting Reform in the Public Sector: Mimicry, Fad or Necessity. Expert Comptable Media, p. 31-42, 2006.

CHRISTIAENS, J.; REYNIERS, B.; ROLLÉ, C. Impact of IPSAS on reforming governmental financial information systems: a comparative study. International Review of Administrative Sciences, v. 76, i. 3, p. 537-554, 2010.

CONSELHO FEDERAL DE CONTABILIDADE. Normas Internacionais de Contabilidade para o setor público. 2010. Disponível em: <http://portalcfc.org.br/wordpress/wp-content/uploads/2013/01/ipsas2010_web.pdf>. Acesso em: 15 nov. 2014.

CONSELHO FEDERAL DE CONTABILIDADE. Resolução CFC n. 1.128, de 21 de novembro de 2008. Dispõe da conceituação, objetivo e campo de aplicação. Diário Oficial da União, Brasília, DF, 21 nov. 2008. Disponível em: <http://www2. cfc.org.br/sisweb/sre/detalhes_sre.aspx?Codigo=2008/001128 $>$. Acesso em: 10 set. 2014.

CRANE, D. Invisible colleges: diffusion ok knowledge in scientific communities. London: University of Chicago Press, 1972.

CRESWELL, J. W. Research design: qualitative \& quantitative approaches. Thousand Oaks: Sage, 1994. 
DARÓS, L. L.; PEREIRA, A. S. Análise das normas brasileiras de contabilidade aplicadas ao setor público - NBCASP: mudanças e desafios para a contabilidade pública. In: CONGRESSO USP DE CONTROLADORIA E CONTABILIDADE, 2009, São Paulo. Anais... São Paulo, 2009.

DOBIJA, M. Public Sector Accounting: Concepts, Methods, Contingencies. The International Journal of Accounting, v. 35, n. 4, p. 553-571, 2000.

DYBALL, M. C.; POULLAOS, C.; CHUA, W. F. Accounting and empire: professionalization-as-resistance: the case of Philippines. Critical Perspectives on Accounting, v. 18, i. 4, p. 415-449, 2007.

DYKXHOORN, H. J.; SINNING, K. E. Perceptions of master of accountancy graduates concerning their job search and employment experiences with public accounting firms. Journal of Accounting Education, v. 14, i. 4, p. 415-434, 1997.

DYKXHOORN, H. J.; SINNING, K. E. Should the accounting profession fear greater government regulation? A look at the German profession. Journal of International Accounting, Auditing and Taxation, v. 1, i. 1, p. 81-91, 1992.

EDWARDS, J. R.; ANDERSON, M.; CHANDLER, R. A. Claiming a jurisdiction for the "Public Accountant" in England prior to organisational fusion. Accounting, Organizations and Society, v. 32, i. 1, p. 61-100, 2007.

EUNNELL, W. N. Pathological responses to accounting controls: The British commissariat in the Crimea. Critical Perspectives on Accounting, v. 1, i. 4, p. 319-335, 1990.

FERREIRA, A. B.; MARCHESINI, C. F. A contabilidade pública em face da padronização contábil. Revista Científica Semana Acadêmica, v. 1, n. 5, p. 1-14, 2011.

FUNNELL, W. N. Pathological responses to accounting controls: The British commissariat in the Crimea. Critical Perspectives on Accounting, v. 1, i. 4, p. 319-335, 1990.

GE, J.; LIN, Z. J. Economic reforms and accounting internationalization in the People's Republic of China. Journal of International Accounting, Auditing and Taxation, v. 2, i. 2, p. 129-143, 1993.

GLYNN, J. J. Public sector accounting \& financial control. Critical Perspectives on Accounting, v. 15, i. 2, p. 3-25, 1990. 
GODDARD, A. Contemporary public sector accounting research-An international comparison of journal papers. The British Accounting Review, v. 42, i. 2, p. 75-87, 2010.

GORDON, T. et al. A comparative empirical examination of extent of disclosure by private and public colleges and universities in the United States. Journal of Accounting and Public Policy, v. 21, i. 3, p. 235-275, 2002.

GRATERON, I. R. G. Auditoria de gestão: utilização de indicadores de gestão no setor público. Caderno de Estudos, n. 21, p. 1-18, 1999.

GROSSI, G.; SOVERCHIA, M. European Commission adoption of IPSAS to reform financial reporting. Abacus, v. 47, i. 4, p. 525-552, 2011.

HARDIN, J. R.; STOCKS, M. H.; GRAVES, O. F. The effect of match or mismatch between the career anchors and the job set tings of CPAs: An empirical analysis. Advances in Accounting, v. 18, p. 119-148, 2001.

HODGES, R.; MELLETT, H. The UK private finance initiative: an accounting retrospective. The British Accounting Review, v. 44, i. 4, p. 235-247, 2012.

HOOD, C. The "New Public Management” in the 1980s: variations on a theme. Accounting, organizations and society, v. 20, i. 2, p. 93-109, 1995.

HOQUE, Z. Using journal articles to teach public sector accounting in higher education. Journal of Accounting Education, v. 20, i. 3, p. 139-161, 2002.

HUMPHREY, C. Reflecting on attempts to develop a financial management information system for the probation service in England and Wales: some observations on the relationship between the claims of accounting and its practice. Accounting, Organizations and Society, v. 19, i. 2, p. 147-178, 1994.

HYNDMAN, N.; CONNOLLY, C. Accruals accounting in the public sector: A road not always taken. Management Accounting Research, v. 22, i. 1, p. 36-45, 2011.

HYNDMAN, N.; EDEN, R. A study of the coordination of mission, objectives and targets in UK executive agencies. Management Accounting Research, v. 11, i. 2, p. 175-191, 2000.

ILIE, E.; MIOSE, N. M. IPSAS and the application of these standards in the Romania. Procedia-Social and Behavioral Sciences, v. 62, p. 35-39, 2012. 
INTERNATIONAL FEDERATION OF ACCOUNTANTS. International Public Sector Accounting Standards Board (IPSAS), Cash Basis. Financial Reporting Under the Cash Basis of Accounting. New York: International Federation of Accountants, 2003.

INTERNATIONAL FEDERATION OF ACCOUNTANTS. Public Sector Committee. About-ipsasb. Disponível em: <http://www.ifac.org/public-sector/about-ipsasb>. Acesso em: 15 dez. 2014.

INTERNATIONAL PUBLIC SECTOR ACCOUNTING STANDARDS BOARD. Handbook of International Public Sector Accounting Pronouncements. New York: International Federation of Accountants (IFAC), 2014. v. 1.

INTERNATIONAL PUBLIC SECTOR ACCOUNTING STANDARDS BOARD. Handbook of International Public Sector Accounting Pronouncements. New York: International Federation of Accountants (IFAC), 2014. v. 2.

JESUS, M. A. J.; EIRADO, J. S. B. Relevance of accounting information to public sector accountability: A study of Brazilian federal public universities. Tékhne, v. 10, i. 2, p. 87-98, 2012.

JONES, R.; PENDLEBURY, M. Public sector accounting. 6. ed. Inglaterra: Pearson Education, 2010.

LIMA, D. V.; CASTRO, R. G. Contabilidade pública: integrando União, Estados e Municípios (Siafi e Siafem). 3. ed. 5. reimpr. São Paulo: Atlas, 2012.

LIMA, D. V.; GUEDES, M. A.; SANTANA, C. M. As Normas Brasileiras de Contabilidade aplicadas ao setor público e a legislação contábil pública brasileira: uma análise comparativa à luz da teoria contábil. Contabilidade, Gestão e Governança, v. 12, n. 2, 2009.

LOPES, J. E. G. et al. Um estudo sobre a complementaridade do fluxo de caixa e do balanço financeiro após a aprovação da NBCASP 16.6 para o setor público. In: CONGRESSO DA ASSOCIAÇÃO NACIONAL DE PROGRAMAS DE PÓS-GRADUAÇÃO EM CIÊNCIAS CONTÁBEIS, 8., Natal, 2010. Anais eletrônicos... Natal, 2010. Disponível em: <http://congressos.anpcont.org.br/congressos-antigos/iv/images/cue_83.pdf>. Acesso em: 20 out. 2014. 
LUNARDI, M. S.; CASTRO, J. M. F. Visualização dos resultados do Yahoo em nuvens de texto: uma aplicação construída a partir de web services. InfoDesign Revista Brasileira de Design da Informação, v. 5, n. 1, p. 21-35, 2008.

MACÊDO, F. F. R. R.; KLANN, R. C. Análise das Normas Brasileiras de Contabilidade Aplicadas ao Setor Público (NBCASP): um estudo nas Unidades da Federação do Brasil. Revista Ambiente Contábil, v. 6, n. 1, p. 253-272, 2014.

MACÊDO, J. M. A. et al. Convergência contábil na área pública: uma análise das percepções dos auditores de TCES, contadores e gestores públicos. Revista de Contabilidade e Organizações, v. 4, n. 8, p. 6991, 2010.

MACIAS-CHAPULA, C. A. O papel da informetria e da cienciometria e sua perspectiva nacional e internacional. Ciência da Informação, v. 27, n. 2, p. 134-140, 1998.

MARTES, A. C. B. et al. Fórum-redes sociais e inter organizacionais. Revista de administração de empresas, v. 46, n. 3, p. 10-15, 2006.

MELO, K. B.; PRIETO, M. F.; ANDRADE, M. E. M. C. Convergência das Normas Brasileiras Aplicadas ao Setor Público: um estudo exploratório no município de Uberlândia-MG. Revista de Contabilidade da UFBA, v. 8, n. 2, p. 21-35, 2014.

MENDONÇA, T. S.; MACHADO, D. P. A Contabilidade Pública e a convergência aos padrões internacionais. In: SALÃO INTERNACIONAL DE ENSINO, PESQUISA E EXTENSÃO, 2., 2010, Bagé. Anais... Bagé: Unipampa, 2010.

MORAIS, L. M.; PLATT NETO, O. A. A reforma contábil promovida pelas NBCASP e o processo de convergência: implicações e perspectivas. In: CONGRESSO ASSOCIAÇÃO NACIONAL DOS PROGRAMAS DE PÓS-GRADUAÇÃO EM CIÊNCIAS CONTÁBEIS, 5., 2011, Vitória. Anais eletrônicos...Vitória, 2011. Disponível em: <http://congressos.anpcont.org.br/congressos-antigos/v/images/164-2. pdf>. Acesso em: 10 out. 2014.

NOGUEIRA, S. P.; JORGE, S. M. Adequacy of the local government financial reporting model in the context of internal decision-making: an exploratory study in the municipality of Bragança. Tékhne, v. 10, i. 2, p. 74-86, 2012.

OLIVEIRA, R. R.; SILVA, A. M. C.; MORAES, M. C. C. Transparência do orçamento governamental dos países: um estudo acerca da associação entre IAO, IDH, PIB e IPSAS. Pensar Contábil, v. 10, n. 42, 2008. 
OULASVIRTA, L. The reluctance of a developed country to choose International Public Sector Accounting Standards of the IFAC. A critical case study. Critical Perspectives on Accounting, 2012.

PICCOLI, M. R.; KLANN, R. C. A percepção dos contadores públicos em relação às Normas Brasileiras de Contabilidade Aplicadas ao Setor Público-NBCASP. Revista do Serviço Público, v. 66, n. 3, p. 425-448, 2015.

PINA, V.; TORRES, L.; ACERETE, B. Are ICTs promoting government accountability?: A comparative analysis of e-governance developments in 19 OECD countries. Critical Perspectives on Accounting, v. 18, n. 5, p. 583-602, 2007.

PINTO, A. L. et al. Indicadores científicos na literatura em bibliometria e cientometria através das redes sociais. Brazilian Journal of Information Science (BJIS), São Paulo, v. 1, n. 1, p. 58-76, 2007. Disponível em: <www.bjis.unesp.br>. Acesso em: 15 jan. 2015.

REZENDE, A. J.; SLOMSKI, V.; CORRAR, L. J. A gestão pública municipal e a eficiência dos gastos públicos: uma investigação empírica entre as políticas públicas e o índice de desenvolvimento humano (IDH) dos municípios do Estado de São Paulo. Revista Universo Contábil, v. 1, n. 1, p. 24-40, 2005.

RIBEIRO, C. M. A.; BIZERRA, A. L. V. Convergência contábil na administração pública federal: o caso das Instituições Federais de Ensino do Estado do Rio de Janeiro. Pensar Contábil, v. 13, n. 52, 2011.

RIBEIRO FILHO, J. F. et al. Evolução da contabilidade financeira na perspectiva emancipatória de Erich Fromm: o processo de construção das Normas Brasileiras de Contabilidade Aplicadas ao Setor Público -NBCASP. Sociedade, Contabilidade e Gestão, v. 4, n. 1, 2010.

ROSSI, N.; TREQUATTRINI, R. IPSAS and accounting systems in the Italian public administrations: expected changes and implementation scenarios. Journal of Modern Accounting and Auditing, v. 7, i. 2, p. 134-147, 2011.

\section{SECRETARIA DO TESOURO NACIONAL. Manual de contabilidade aplicado} ao setor público. 6. ed. 2015. Disponível em: <http://www.tesouro.fazenda.gov.br/ mcasp>. Acesso em: 12 dez. 2014.

SILVA, L. M. Contabilidade governamental: um enfoque administrativo da nova contabilidade pública. 9. ed. São Paulo: Atlas, 2011. 
SLOMSKI, V. Controladoria e governança na gestão pública. São Paulo: Atlas, 2005.

SMITH, K. J.; AHADIAT, N. Empirical evidence on factors significant for employment in government accounting. Journal of Accounting Education, v. 13, i. 1, p. 59-74, 1996.

SOUZA, R. G. et al. O regime de competência no setor público brasileiro: uma pesquisa empírica sobre a utilidade da informação contábil. Revista Contabilidade \& Finanças, v. 24. n. 63, p. 219-230, 2013.

TOUDAS, K.; POUTOS, E.; BALIOS, D. Concept, regulations and institutional issues of IPSAS: a critical review. European Journal of Business and Social Sciences, v. 2, i. 1, p. 43-54, 2013.

VICENTE, E. F. R; MORAIS, L. M.; PLATT NETO, O. A. A reforma na contabilidade pública brasileira e o processo de convergência: implicações e perspectivas. RIC - Revista de Informação Contábil, v. 6, n. 2, p. 1-20, 2012.

ZEFF, S. A. International Research in Public Sector Accounting, Reporting, and Auditing: edited by Vicente Montesinos and José Manuel Vela, Instituto Valenciano de Investigaciones Económicas, SA València, Spain, 1995, 308 pp. The International Journal of Accounting, v. 35, i. 2, p. 292, 2000.

WASSERMAN, S.; GALASKIEWICZ, J. Advances in social network analysis: research in the social and behavioral sciences. London: Sage, 1994.

Como citar este artigo:

\section{ABNT}

MORÁS, Vânia Regina; KLANN, Roberto Carlos. Contabilidade do setor público: um estudo das redes sociais publicadas em periódicos internacionais. RACE, Revista de Administração, Contabilidade e Economia, Joaçaba: Ed. Unoesc, v. 15, n. 3, p. 1143-1168, set./dez. 2016. Disponível em: <http://editora.unoesc.edu.br/index. php/race>. Acesso em: dia/mês/ano.

\section{APA}

Morás, V. R., \& Klann, R. C. (2016). Contabilidade do setor público: um estudo das redes sociais publicadas em periódicos internacionais. RACE, Revista de Administração, Contabilidade e Economia, 15(3), 1143-1168. Recuperado em dia/mês/ano, de http://editora.unoesc.edu.br/index.php/race 